\title{
LA INCLUSIÓN DEL SUFRIMIENTO PSÍQUICO: UN DESAFIO PÀRA LA EPIDEMIOLOGIA
}

Ana Cecília Augsburger Universidad Nacional de Rosario

RESUMEN: El artículo indaga en qué medida los criterios y las categorías utilizadas para el diagnóstico y la clasificación de los problemas de salud mental, constituyen un obstáculo teórico y metodológico para el desarrollo de la epidemiología en salud mental. Los resultados contemplan dos aspectos. (1)Una reflexión teórica destinada a redefinir el objeto de estudio que históricamente la disciplina construyó para sí, analizando de manera crítica las modalidades clasificatorias para los problemas psíquicos. (2)Un trabajo de campo en el que se analizan los esquemas teóricos y operativos en los que intervienen los profesionales de la salud mental. Se busca conocer en qué medida tanto las nociones más empíricas, como la formación científica que reciben los psicólogos se adecuan a las categorías propuestas para identificar y diagnosticar las patologías psíquicas (CIE ó DSM). Si el sufrimiento humano no es sólo un problema de orden biológico, sino que es producido en contextos culturales y socio históricos definidos, es necesariamente crítica la mirada sobre sistemas clasificatorios que no contemplan las dimensiones sociales, culturales o institucionales.

PALABRAS-CLAVE: Salud mental / Epidemiología / Clasificación de enfermedades/ Diagnóstico/ Sufrimiento psíquico.

\section{THE INCLUSION OF MENTAL SUFFERING: A CHALLENGE FOR EPIDEMIOLOGY}

ABSTRACT:The article examines in what extend the criteria and the categories used for diagnosis and the classification of mental health problems, are actually theoretical and methodological obstacles for the development of Epidemiology in mental health. The results lead to two main issues: (1) A theoretical consideration pointing to re-define the object of study traditionally built by the discipline itself, analyzing the classificatory schemes for psychics problems. (2) A work in the field analyzing the theoretical and operative schemes in which the professionals in mental health intervene. It tries to clarify in what extend the empirical notions and the scientific skills received by the psychologists, are the correct ones to the categories proposed to identify and diagnose the psychic pathologies. If human suffering is not just a biological problem, but takes place in a social, historical and cultural environment, it is necessary to have a very critical eye on the classificatory systems that ignore the social, cultural and institutional dimensions.

KEY-WORDS: Mental health. Epidemiology. Classification of illnesses. Diagnosis. Psychic suffering.

\section{INTRODUCCION}

El articulo propone argumentar y debati acerca de un problema que se identifica como obstaculizador para el desenvolvimiento de la epidemiologia en salud mental. La investigación epidemiológica está fundada sobre una clasificación internacionalmente aceptada de enfermedades o problemas de salud, cuya organización refiere a un sistema de categorías a las que se les asignan entidades mórbidas de acuerdo a criterios pre-establecidos. Sin embargo, los criterios o las lógicas con que se clasifican los problemas atinentes a la salud psíquica se ubican como un ámbito de controversias teóricas y operativas cuyas consecuencias repercuten tanto en la capacidad técnica de darle visibilidad y recepcionar a los procesos de padecimiento psíquico de los diversos grupos de población, como en formular un juicio calificador para dichos problemas.

Las reflexiones y resultados de este trabajo son producto de una investigación destinada a establecer los obstáculos y las limitaciones presentes para la producción de información epidemiológica sobre la salud mental de la población en el contexto particular del municipio de Rosario (Argentina). Con la finalidad de lograr una comprensión exhaustiva sobre las características que el problema planteado adquiere en el contexto local se decidió tratar al mismo como un estudio de casos. El estudio de casos nos permite 
Augsburger; A.C. "La inclusión del sufrimiento psíquico: un desafio para la epidemiologia"

pensar el municipio como una unidad específica y compleja, respondiendo a los requerimientos de un abordaje que no suprima lo singular. Reconocer lo que el caso tiene de particular, a través de conocerlo con profundidad, no obsta para que se hayan podido establecer relaciones con niveles de mayor generalidad en el problema planteado, que nos remiten al debate en torno de las categorias y criterios con que la epidemiología clasifica los problemas de la salud mental.

La profundización sobre los modos como se perciben, categorizan y diagnostican los problemas de salud mental presenta dos aspectos cuya especificidad permite analizarlos separadamente, pero que, no obstante, se encuentran en una estrecha relación. El primer aspecto está ligado a la definición del objeto que la epidemiología en salud mental escoge para sí. Adoptar la noción de enfermedad mental supone una serie de limitaciones para reconocer e identificar situaciones de padecimiento que no son homologables a las entidades patológicas, sumándose a ello la disparidad y la impugnación a los criterios señalados para su diagnóstico. Es en función de este nudo problemático que se analizan criticamente las clasificaciones internacionales de enfermedades.

El segundo aspecto remite a la indagación sobre los esquemas teóricos y operativos con los que intervienen en sus prácticas los profesionales de la salud mental. Si existen criterios preestablecidos (Clasificación internacional de enfermedades ó Manual diagnóstico y estadístico de los trastornos mentales) para identificar y diagnosticar las patologías psíquicas, es menester interrogar en qué medida tanto las nociones más empíricas como la formación científica que reciben los psicólogos se adecuan a las categorías propuestas.

Las transformaciones y situaciones de crisis que atraviesan tanto la vida societal como la cotidianidad en el ámbito familiar son espacios de producción de condiciones críticas que contribuyen a la generación de sufrimiento psíquico, (GALENDE, 1997, 2000; LUDERMIR, 2000; PALACIOS, 2002; MINAYO-GOMEZ, 2002) requiriendo categorías que describan éste adecuadamente, sin considerarlo necesariamente en términos de enfermedad.

LAS TAXONOMIAS DE LOS PROBLEMAS DE SALUD MENTAL: UN AMBITO DE CONTROVERSIAS

El proceso de clasificación de las enfer- medades mentales es un componente necesario en el que se funda la investigación epidemiológica en el campo de la salud mental. Una clasificación de enfermedades se define como un sistema de categorías a las cuales se asignan entidades mórbidas de acuerdo con criterios pre-establecidos. El propósito que da origen a la clasificación internacional de enfermedades es permitir el registro sistemático, el análisis, la interpretación y la comparación de los datos de mortalidad y morbilidad recolectados en diferentes países o áreas, así como en distintos momentos históricos.

En el dominio específico de los problemas de la salud mental las normatizaciones con mayor desarrollo y difusión se organizan sobre una descripción taxonómica de las enfermedades mentales que toma por base la nosografía psiquiátrica. Tal es el caso del Capítulo V de la Décima clasificación internacional de enfermedades y problemas relacionados con la salud, que reúne los llamados trastornos mentales y del comportamiento, o bien posterior en el tiempo, la clasificación elaborada por la Asociación Americana de Psiquiatría, (DSM) que ha sufrido continuas renovaciones. La clasificación estadística de enfermedades (1995), que se constituye como un eje referencial ineludible a nivel internacional, adopta para los problemas de salud mental la categoría de trastorno mental. Confirma allí la multiplicidad de significados con los que éste es comprendido: malestar, descontrol, limitación, incapacidad, patrón sindrómico, etc. Cada uno de ellos puede ser un indicador útil para un tipo de trastorno mental, pero ninguno equivale al concepto. A nivel internacional la mayoría de los estudios epidemiológicos revisados organizan su propuesta clasificatoria a partir del concepto de enfermedad mental, tomando como base algunos de los sistemas hegemónicos mencionados (CIE ó DSM). Argentina no se distingue de esa situación, salvo por la carencia casi absoluta de estudios epidemiólogicos que indaguen la situación de salud mental de sus grupos poblacionales. A principios de los años '80, se lleva a cabo el único estudio de carácter descriptivo a escala nacional que toma por eje la prevalencia poblacional de patologías mentales en la República Argentina, adoptando la Clasificación Internacional de Enfermedades, y un instrumento estandarizado para la formulación del diagnóstico, el Examen del Estado Presente, (P.S.E.). (CASULLO; ASZKENAZI, 1985).

La revisión y análisis de un conjunto de investigaciones epidemiológicas muestra a nivel internacional la década del '70 como el momento en el cual se produce un viraje en torno de la 
producción epidemiológica en salud mental. El corte temporal delimita líneas de investigación con énfasis diferenciales. La primera de ellas, que se enmarca dentro de una posición cercana al relativismo cultural y el rescate de lo microsocial, tiene por eje la comprensión de las condiciones sociales que intervienen en la producción de las enfermedades mentales.

La segunda, posterior en el tiempo, está orientada por la búsqueda de parámetros de objetividad universal y comparabilidad de los resultados, a través de estudios extensivos con herramientas de screenning poblacional. Es esta última línea la que encuentra al momento actual mayor desarrollo contando con el apoyo pleno de organizaciones internacionales (KESSLER, 2000; OMS, 2000).

Los avances producidos como consecuencia de esos cambios en los últimos 20 años son de gran relevancia permitiendo orientar esfuerzos en el desenvolvimiento de instrumentos de identificación de casos con mayor poder discriminativo. La obtención de diagnósticos fiables y válidos con la utilización de los nuevos instrumentos dio la posibilidad de aplicar encuestas epidemiológicas a grandes grupos poblacionales y en un número diverso de países, propiciando el análisis comparativo de los datos obtenidos (KESSLER, 2000). Sin embargo, y reconociendo éstos avances de la epidemiología psiquiátrica, algunos de los elementos planteados permiten visualizar, a nuestro juicio, ciertos obstáculos en el abordaje epidemiológico de los problemas de salud mental. La utilización de las clasificaciones internacionales promueve que el análisis del estado de salud mental en los grupos de población se focalice en la categoría de enfermedad mental. Sistemas que, organizados con una perspectiva nosográfica, muestran dificultad para identificar aquellas expresiones de sufrimiento psíquico que no se encuadran como enfermedad objetivada.

La adopción para la definición del concepto de «enfermedad mental» de una visión a-histórica, y con pretensiones de universalidad, en función de incrementar la objetividad del conocimiento para permitir la comparabilidad de los resultados se encuadra en una epistemología de corte positivista. La nosografía psiquiátrica, con base en ese paradigma, ha trabajado con un concepto de enfermedad positiva, considerando como tal sólo aquello que se puede ver y comprobar porque produce señales y síntomas; lo que conlleva a desacreditar la enunciación de malestar por parte de los sujetos si no se acompaña de síntomas discernibles desde el saber científico - técnico. Así,
Psicologia \& Sociedade; 16 (2): 71 -80; maio/ago.2004

la enfermedad aparece considerada como una entidad natural que responde en su génesis a mecanismos de causalidad mecánica, predominantemente biológicos, consolidando un modelo de pensamiento que no permite problematizar en el campo epidemiológico los complejos procesos sociales e históricos que determinan formas singulares y colectivas diferenciadas de vivir, padecer y enfermar.

Adoptando una perspectiva crítica con este horizonte de racionalidad epidemiológica, señalamos que la utilización de un concepto universal para la enfermedad mental opaca el alto grado de producción y designación cultural y social que presentan los llamados problemas psíquicos. Son ejemplos válidos en esta dirección la categoría diagnóstica de retraso mental (MUEL, 1975), ó la de homosexualidad (CONRAD, SCHNEIDER; 1982) que como estados o condiciones de las personas, han encontrado variaciones históricas en su significación y adquirido o no la designación de enfermedad o trastorno mental según parámetros culturales o valoraciones sociales cambiantes en el tiempo.

El enfoque prioritario que adopta la epidemiología psiquiátrica, (KESSLER, 2000) centrada en la estimación de las prevalencias y en la clasificación del tipo de trastorno produce una mirada reducida puesto que evalúa el estado de salud mental de las poblaciones con la presencia o ausencia de enfermedad psíquica, sin incorporar miradas más positivas de la salud ni tampoco elementos vinculados a la calidad de vida de esos grupos poblacionales, que dificilmente pueden expresarse en términos de enfermedad.

Finalmente, el interés depositado en formular resultados comparables para grandes grupos poblacionales conduce a aceptar la población como una categoría natural, como un denominador epidemiológico homogéneo. La crítica a esta perspectiva epidemiológica generalizante es vehiculizada a través de los conceptos de historicidad, heterogeneidad, y contradicción, categorías éstas que dan cuenta de los atributos necesarios para describir, distinguir y diferenciar a los grupos sociales.

\section{METODOLOGIA}

El municipio de Rosario (Argentina) es una ciudad industrial localizada en la Provincia de Santa Fé, que tiene alrededor de un millón de habitantes y cuenta con un Sistema de Salud Pública dependiente de la administración municipal compuesto por una red de instituciones prestadoras 
Augsburger; A.C. "La inclusión del sufrimiento psíquico: un desafio para la epidemiologia"

de servicios con diversos grados de complejidad. La red de salud tiene por objeto dar cobertura sanitaria a la población residente en el municipio, pero su área de influencia abarca una región geográfica más amplia que trasciende los límites de la ciudad. Cuenta con una amplia infraestructura dotada de un conjunto de hospitales con especificidades diversas y que incluyen servicios de alta complejidad, dos maternidades que recepcionan casi el $80 \%$ de los partos del sector público, un Centro de Especialidades Médicas Ambulatorias, que recepciona población referenciada y 45 centros de salud, distribuidos en el municipio y organizados a través de la dirección de Atención Primaria de la Salud.

El proceso de investigación se orientó a fortalecer la producción de información epidemiológica de salud mental en el municipio de Rosario, evaluando las posibilidades y los obstáculos para la adopción de una taxonomía de los problemas de salud mental a nivel local. Con ese marco se escoge un abordaje de índole cualitativo que permitiera indagar en el universo de significaciones, valoraciones y prácticas que sostienen los trabajadores de la salud mental; reconocer los marcos teóricos y operativos que organizan referencialmente tanto la percepción y comprensión de los procesos de salud - enfermedad mental como la elaboración y formulación de juicios diagnósticos.

\section{MATERIAL, SUJETOS Y PROCEDIMIENTOS}

En el trabajo de campo destinado a la recolección de información empírica, se combinaron entrevistas semi estructuradas a los profesionales psicólogos, con el análisis pormenorizado de los registros de consulta de las prácticas asistenciales cotidianas. Fueron entrevistados 20 profesionales psicólogos dependientes del Programa de Salud Mental del Sistema de Salud Pública del municipio de Rosario (Argentina). Las entrevistas se realizaron en los ámbitos de trabajo, centros de salud y consultorios hospitalarios, en una o más sesiones y con una duración aproximada de 2 horas.

Atendiendo al carácter cualitativo del trabajo no se utilizaron técnicas de muestreo sino que los profesionales se seleccionaron por criterios a partir de la definición conceptual y operacional de unidades de estudio (GRIMBERG, 1999), según los siguientes componentes: institución de trabajo, años de graduación y adscripción teórica reconocida.

Si bien los ejes temáticos seleccionados en el transcurso de la indagación han sido de mayor amplitud, se han privilegiado en la exposición de los resultados dos categorías sustanciales en torno a los marcos conceptuales e ideológicos que orientan las prácticas profesionales: el proceso de salud enfermedad mental, y la racionalidad utilizada para la construcción de los diagnósticos.

\section{RESULTADOS}

La perspectiva de los profesionales psicólogos sobre el proceso de salud - enfermedad mental.

A lo largo de la historia los grupos sociales han sistematizado modalidades propias de conocimiento sobre la salud y la enfermedad, configurando un conjunto de nociones y representaciones sobre esos objetos. Esos conocimientos y concepciones, propios de un momento histórico particular, organizan tanto un saber popular, resultado de prácticas cotidianas, como un cuerpo teórico e ideológico entramado a una práctica profesional específica. En el campo de la producción científica se acumulan desarrollos muchas veces contradictorios o antagónicos. Los obstáculos teóricos para avanzar en una conceptualización de la salud y de la enfermedad son señalados por diferentes autores, que destacan a su vez la multivocidad de significaciones que les son asignadas. (BERLINGUER, 1993; ALMEIDA FILHO, 1994; GRMEK, 1995)

Sobre éstas limitaciones teóricas se inscriben las dificultades que los profesionales psicólogos sitúan para delimitar la salud y la enfermedad mental.

"No sé que es la salud..." “ ....yo recuerdo una definición de O.P.S. que decía algo del bienestar físico, mental y social...", dirá alguno de los entrevistados. (Ps. 7)

"Los parámetros de la salud y de la enfermedad son distintos a los que plantea el discurso médico. Yo no sé si podría (en el marco de la salud mental) hablar de que alguien está enfermo o está sano". (Ps. 16)

"Tomando en cuenta que nosotros no tenemos diagnósticos precisos como sí tiene la medicina o la psiquiatría, no podría definir las consultas en términos de enfermedad". (Ps.5)

Desde esta perspectiva, la categoría enfermedad parece más adecuada para el ámbito de la medicina, en virtud de contar con delimitaciones diagnósticas precisas de las cuales derivan estrategias o consensos terapéuticos. La 
objetividad con que operan los criterios médicos para designar una enfermedad no es reconocida como posible en el campo de la salud mental. Sin embargo, no hay en su interior un proceso de reformulacioón de tal categoría.

La fragmentación entre los conceptos y la experiencia se hace presente cuando se interroga a los profesionales acerca de si los pacientes que consultan podrían ser descriptos como personas enfermas. Los profesionales, que excluyen la categoría de enfermedad de sus presupuestos teóricos, van a reconocer, por el contrario, situaciones concretas en las que determinados pacientes sí son identificados como enfermos. Estos especialistas ratifican la presencia de una enfermedad frente a estados de salud de algunas personas concretas.

"Con relación a la enfermedad men-

tal, acá hay muy pocas consultas de psicosis. Una mujer a la que atendieron en la guardia y yo fui a ver..." (Ps. 9)

"En el caso de alguien que tiene una psicosis sí diría que esa persona está enferma. Y en el caso de la perversión también. (Ps.3)

"A lo mejor con un diagnóstico de anorexia yo podría decir que hay una enfermedad..." (Ps. 12)

La relación entre las formulaciones teóricas y las prácticas no es lineal, sino que constituyen ambas un conjunto abierto con elementos contradictorios y espacios de imprecisión. Resulta una tarea más sencilla distinguir e identifcar con la observación y la experiencia a determinado evento como patológico, que conceptualizar la enfermedad en una dimensión de mayor abstracción. Determinados fenómenos se interpretan como enfermedades en la medida que se presentan en forma concreta en personas, sin que ello implique el reconocimiento de un objeto de construcción teórica, o aún cuando contradiga algunas de las proposiciones formuladas.

El intento de producir un ordenamiento clasificatorio para los problemas de salud mental muestra que definir e identificar la enfermedad mental es una tarea compleja. Los problemas de orden psíquico o mental no se ajustan al modelo de razonamiento causal que la clínica asume para definir los fenómenos patológicos, ni se encuadran dentro de la perspectiva semiológica que relaciona con un significado unívoco síntomas y signos a un evento patológico. Cuando la perspectiva gnosográfica clásica delimita el universo de entidades mórbidas a todas aquellas señales, signos o síntomas factibles de verse o comprobarse, excluye padecimiento. En esa perspectiva la dimensión subjetiva del sufrimiento es una dimensión ausen-

En la visión de los psicólogos entrevistados, cuya práctica se enmarca mayoritariamente en la teoría psicoanalítica, el sufrimiento humano designa el objeto que otorga especificidad a su quehacer. Para ellos, son las situaciones de sufrimiento las que inducen de manera prioritaria a alguien a solicitar ayuda.

«Las personas vienen por que sufren, sienten que no pueden resolverlo solos y deciden consultar». (Ps. 17) «Lo que distingue a las consultas de salud mental es el sufrimiento, no la enfermedad». «Las personas consultan porque sufren. Sufren, y el sufrimiento... iba a decir psíquico... Si, creo que me atrevo a decir sufrimiento psíquico.» (Ps. 15)

«Vienen por algo que les molesta, preocupaciones. En el caso de niños, que dicen mi hijo es bárbaro, no entiendo porqué. Le pasa esto, o se orina encima y tiene nueve años. Hay alguna interpelación ahí, y de algún modo un sufrimiento.» (Ps.2)

La posibilidad de trabajar en el campo de la salud mental con la noción de sufrimiento psíquico o de malestar encuentra el apoyo de diversos autores, (FREUD, 1930/1981; GALENDE, 1997; BURIN, 1991), cuyas producciones podrían contribuir en el terreno epidemiológico a superar algunas de las limitaciones que se han señalado.

En nuestra perspectiva, la incorporación del concepto de sufrimiento psíquico y su distinción de la patología mental permite ubicar dos cuestiones específicas de los problemas atinentes a la salud mental. Por un lado, evita considerar los conflictos que devienen de la vida cotidiana y de las interrelaciones sociales, en términos de patologías. Las condiciones concretas en que se generan los padecimientos le otorgan a éstos un carácter procesual e histórico que no queda expresado en las clasificaciones mórbidas; y cuya utilización conduce a una patologización de las situaciones cotidianas. Asimismo, la emergencia del sufrimiento psíquico no conduce necesariamente a la enfermedad, puede tanto precederla como ser divergente de ella.

Por otro lado, el sufrimiento permite incorporar la dimensión subjetiva del padecimiento, dimensión sin duda ausente en la gnosografía clásica. Los trabajos y experiencias tanto de Freud 
Augsburger; A.C. "La inclusión del sufrimiento psíquico: un desafio para la epidemiologia"

(1930/1981) como de Clavreul (1983) o de Raimbault (1985), muestran cómo el avance del conocimiento sobre el cuerpo humano y de sus formas de reparación no puede sustituir la percepción y expresión de un sufrimiento que se rige con coordenadas distintas de las que marca la biología, y que remiten al plano de la constitución desiderativa de los sujetos así como a sus procesos de constitución cultural y social.

EL DIAGNOSTICO EN SALUD MENTAL. UN PROBLEMA HEREDADO EN EL CAMPO EPIDEMIOLOGICO.

La más marcada expresión del lazo que une la clínica y la epidemiología se revela en la construcción y selección del propio objeto de la epidemiología, dado que la identificación de enfermos en poblaciones es producida, en última instancia, por el abordaje clínico, como lo señala Almeida Filho (1992). Para este autor, el objeto de conocimiento delimitado originariamente por vía de la clínica médica es trasladado prácticamente sin mediaciones de un campo al otro, siendo definido como la enfermedad, en cuanto entidad nosográfica establecida por la taxonomía médica. La noción de morbilidad permite el pasaje de la concepción de enfermedad a un nivel poblacional, señalando entonces el punto en que la epidemiología es subsidiaria de la clínica.

En el campo de la salud mental, la formulación de diagnósticos, cuestión eminentemente clínica, presenta problemas epistemológicos y conceptuales asentados sobre las dificultades de precisar la noción de enfermedad que designa. Un autor como Kessler (2000), enrolado dentro de la llamada epidemiología psiquiátrica, señala esta situación afirmando que la epidemiología psiquiátrica ha avanzado cn retraso respecto de otras ramas de la epidemiología a causa de las dificultades que entraña la conceptualización y la medición de los trastornos mentales. Del mismo modo, los padecimientos mentales circunscriben un conjunto de problemas metodológicos particulares en el desarrollo de procedimientos e instrumentos que permitan la identificación operacional de aquello que designan (ANDREOLI, 2000)

Para los profesionales de salud mental de la Secretaría de Salud Pública, población entrevistada en este estudio, la formulación de diagnósticos es una acción reconocida y aceptada. La totalidad de los mismos afirma que, en el transcurso de los procesos de atención de los pacientes, realiza diagnósticos. No distinguen para su elaboración ninguna otra herramienta específica que no sea la entrevista clínica, siendo el diagnóstico incluido como un elemento más que deviene del trabajo clínico y no como un procedimiento específico. Hay coincidencia en señalar las dificultades para realizarlo en un tiempo breve, de modo que sólo la prolongación de los encuentros a través de las entrevistas preliminares o de un tratamiento permite su formulación. “...el diagnóstico no es algo que se pueda hacer en las primeras entrevistas, sino que lleva su tiempo hacerlo." (Ps.15)

"Al diagnóstico lo hago cuando el paciente lleva ya un tiempo de trabajo, sino no". (Ps. 3)

"A mí me cuesta mucho hacer un diagnóstico, y por lo general hay muy pocos pacientes que inician un tratamiento y lo pueden sostener durante un tiempo bastante prolongado...". (Ps.16)

Las expresiones de los profesionales hacen visibles las dificultades que ellos encuentran para su elaboración. Los conceptos utilizados para la asignación de diagnósticos provienen de su formación en el campo del psicoanálisis, tomando como categoría analítica lo que denominan estructuras psíquicas. Se describen entonces las categorías utilizadas como psicosis, neurosis, perversión, neurosis obsesiva, histeria, etc. Los juicios diagnósticos no refieren, en ésta perspectiva teórica a entidades patológicas sino a procesos de estructuración psíquica. La exposición de esos criterios para la formulación de los diagnósticos es ampliamente divergente de las propuestas clasificatorias adoptadas internacionalmente.

"Yo utilizo los diagnósticos de neurosis, psicosis o perversión, por cómo se posiciona el paciente....". (Ps. 7)

“...en el tema de la guardia, con el diagnóstico evaluás si es una descompensación psicótica o no...". (Ps. 9)

"Los diagnósticos en general los hago usando las categorías del psicoanálisis. Si es neurosis, si es paciente de borde...". (Ps. 3)

Las interrogaciones acerca de cómo y para qué se formula un diagnóstico en el campo de la salud mental van a encontrar respuestas claramente diferenciadas si se atiende a la lógica de las propuestas internacionales o a la perspectiva de los trabajadores en estudio. Las taxonomías 
internacionales se inscriben en la línea que continúa el marco disciplinar originario, aquel donde el concepto de diagnóstico es acuñado, que remite a la medicina mental y posteriormente a la psiquiatría. Las posiciones teóricas de los profesionales entrevistados se enmarcan en los desarrollos del psicoanálisis, perspectiva desde la cual el concepto de diagnóstico es reformulado.

Una de las diferencias centrales respecto a estas dos posiciones se asienta en la inclusión o no de la referencia al sujeto. La apelación al sujeto que puede ser formulada en una doble dirección, tanto con relación al paciente, como a quien formula el juicio diagnóstico.

Desde la perspectiva médico - psiquiátrica el proceso diagnóstico es un procedimiento objetivo que deja por fuera a la persona que lo realiza. Se propone como la tarea de incluir un estado de salud determinado dentro de una entidad gnosográfica a partir de signos y síntomas objetivos. El psicoanálisis, en cambio, sostiene que la elaboración de un diagnóstico incluye inevitablemente a quien lo realiza, en este caso la persona del analista. Desde la perspectiva psicoanalítica el diagnóstico es una dimensión ineludible de la cura que conduce a retomar la indagación acerca del proceso de constitución subjetiva, distinguiéndose éste de un estado patológico. Con abundante precisión el psicoanálisis afirma que la elaboración diagnóstica no significa la formulación de un juicio de un individuo sobre otro individuo, sino que se trata de un señalamiento esencialmente relacional, en donde ambos sujetos se hallan incluidos.

El psicoanálisis, en coherencia con sus postulados de destacar y preservar lo que cada sujeto tiene de más singular se propone abordar caso por caso y, se ha manifestado contrario a la elaboración de diagnósticos al modo como los formula la psiquiatría, en tanto es pensado como la inclusión de cada padeciente en una clase gnosográfica predeterminada. Por el contrario, desde la visión taxonómica de la psiquiatría, la consideración de la sintomatología conlleva a que el médico omita aquello que el paciente puede decir de sí mismo. $\mathrm{El}$ especialista debe fijar su atención sobre aquellos elementos necesarios para completar un esquema de síntomas y signos que le permita establecer un diagnóstico.

La significación teórica que adquiere la noción de síntoma es también lo que marca una ruptura entre la clínica psicoanalítica y la clínica psiquiátrica El síntoma es una vía directa para acceder al campo del padecimiento humano, ubicándose generalmente como aquello por lo cual
Psicologia \& Sociedade; 16 (2): 71 -80; maio/ago.2004

se decide una consulta, se busca una ayuda. Para la psiquiatría los síntomas constituyen una evidencia empírica que permiten afirmar la existencia de una enfermedad, o la presencia de un trastorno, representando en este sentido una «invariante semiótica» (FELDMAN, 1995). Para el psicoanálisis los síntomas son una expresión del inconsciente que se inscribe en el conjunto de sus producciones, sueños, chistes, actos fallidos, etc. sin poder establecerse una relación unívoca entre síntomas y patología.

En Psicoanálisis y Psiquiatría (1917/1981)

Freud plantea con claridad lo que constituye la diferencia en la propuesta por él inaugurada en cuanto al modo de concebir el síntoma: en la psiquiatría se liga síntoma y significado, y se lo objetiva en una nomenclatura, en el psicoanálisis se destaca la función significante y su lugar para la interrogación. En este sentido Freud impugna la relación anteriormente establecida entre síntoma y enfermedad (FREUD, 1917/1981).

Así, los profesionales reconocen un conjunto de síntomas, que si bien son expresiones de padecimiento subjetivo, no pueden ser clasificadas por ellos como enfermedades mentales. En este sentido, las opiniones que aportan parecen corresponderse con la ruptura epistemológica que Freud realiza para guiar el psicoanálisis: la disociación de la relación establecida entre síntoma $\mathrm{y}$ enfermedad.

«Los pacientes consultan porque sufren, sienten que no pueden resolver solos sus problemas y deciden consultar».(Ps. 14)

«Con relación a la enfermedad mental, yo no uso mucho esa nomenclatura. A lo suma podría decir si es una neurosis o una psicosis más o menos grave." (Ps.7)

"Yo lo que pienso es que los pacientes tienen rasgos, rasgos de una neurosis obsesiva, rasgos histéricos, aparecen rasgos de muchas cosas. Y esos rasgos que presenta una persona que está en tratamiento no son homologables a una enfermedad». (Ps 17.)

«Yo prefiero hablar de conductas desadaptativas y no de enfermedad».

(Ps. 19)

La indagación acerca de los criterios y las categorías que los profesionales psicólogos utilizan para la fomulación de diagnósticos persigue el propósito de evaluar en qué medida esos juicios diagnósticos son pasibles de constituirse en información valida para uso epidemiológico. La 
Augsburger; A.C. "La inclusión del sufrimiento psíquico: un desafio para la epidemiologia"

validez es un componente esencial a la hora de evaluar la calidad de la información producida y su aprovechamiento epidemiológico.

La asimetría existente entre los esquemas referenciales del conjunto de profesionales de la salud mental y los marcos teóricos de construcción de las taxonomías más difundidas da cuenta de un claro obstáculo para el desarrollo de estudios epidemiológicos en el contexto local. Obstáculo que no debe ser mirado sólo en forma restricta al caso del municipio de Rosario en estudio, sino que da cuenta de un conflicto que atañe al espacio de la epidemiología en salud mental como campo de conocimiento.

DISCUSION: ALIMENTANDO EL DEBATE PARA UNA NUEVA TAXONOMIA EN SALUD MENTAL

Fortalecer la construcción de la epidemiología en salud mental para promover un desarrollo que refleje las condiciones y necesidades actuales que este campo presenta, orienta la búsqueda hacia nuevos modelos clasificatorios, problematizando no sólo las taxonomías centradas en entidades mórbidas, sino interrogando y redefiniendo el objeto mismo de estudio. La epidemiología en salud mental utiliza un objeto de estudio definido desde otra disciplina, la clínica psiquiátrica y traslada para sí la categoría de enfermedad mental, apropiándose de un concepto sobre el que existen profundos desacuerdos teóricos y un alto grado de impugnación científica y social. Todos los análisis de prevalencia, incidencia, distribuciones, tasas, etc, que a partir de allí se elaboran, padecen de la limitación de no haber sido precisada la naturaleza misma del objeto "enfermedad mental". Como bien señala Bercherie (1986), la clínica psiquiátrica en su faz descriptiva, ha conseguido realizar un inventario exhaustivo de los diferentes trastornos o problemas psíquicos, pero esa riqueza contrasta con las dificultades e insatisfacciones que en la faz conceptual refieren a la naturaleza misma de la perturbación. En el plano epistemológico se trata de la sumisión del objeto teórico a un fenómeno empírico, de una confusión entre estos dos niveles de indagación científica que produce como consecuencia una falta de precisión de las cualidades del objeto de trabajo.

Cuando la epidemiología en salud mental renuncia a la elaboración de un concepto propio sobre los procesos de salud - enfermedad mental, limita también sus posibilidades de producción de nuevos conocimientos y sobre todo su capacidad de comprender y explicar los problemas de salud mental de los grupos humanos. En éste sentido, tendría mayor riqueza, aun que más lentitud, reconstruir un objeto de estudio propio, que respete tanto la naturaleza singular que presenta la producción y expresión de la subjetividad, como el carácter complejo que atraviesa los problemas de padecimiento psíquíco y que no permite reducirlos a fenómenos orgánicos ni puramente psicológicos, ni puramente sociales.

De ningún modo se trata de desconocer la importancia o la urgencia que puede revestir la patología, pero en el campo de la salud mental se presentan múltiples expresiones que indicando sufrimiento o malestar no pueden encuadrarse en la categoría de enfermedad. Los umbrales de percepción y tolerancia subjetiva y social del dolor psíquico son difíciles de estandarizar a través de parámetros universales, siendo también imprescindible profundizar el debate en torno a los parámetros técnicos que diferencien el punto en que el sufrimiento subjetivo se transforma en enfermedad discernible por terceros. Si se atiende a la especificidad de los problemas de salud mental, la utilización del concepto de sufrimiento psíquico permite destacar y reconocer la dimensión subjetiva que constituye el proceso mismo de enfermar (BERLINGUER, 1994; SUSSER, 1970; BASAGLIA, 1972)

La sujeción acrítica a las taxonomías internacionales vigentes no representa sólo un problema de índole metodológico, sino que tiene consecuencias en las posibilidades concretas de elaboración de nuevos conocimientos. Los resultados hallados en el diagnóstico de la realidad local muestran que los profesionales reconocen el sufrimiento como la expresión de los problemas de salud mental y que ese padecimiento no puede ser decodificado en término de enfermedad.

El interés de avanzar en la producción de información epidemiológica en salud mental en el contexto local, podría orientar la búsqueda hacia aquellas lógicas clasificatorias que no centren su análisis en entidades patológicas. Las nociones más abarcativas de «problema de salud», o de «situación problemática» son una opción a debatir. Uno u otro permitirían incluir situaciones de la vida en familia o de la vida social en general, sin que esas situaciones sean consideradas patologías o disfunciones. Indicar la temporalidad del problema como activo o pasivo, permitiría recuperar su condición de proceso. Y por último, daría lugar a reconocer las facetas subjetivas $\mathrm{u}$ objetivas de los problemas según la ubicación de quién lo evalúa, sean los sujetos afectados, o los profesionales.

La importancia que tiene la generación o 
aceptación de un conjunto clasificatorio es enorme. Las clasificaciones son resultado de los modo como se percibe, se distingue y se organiza un sector determinado de la realidad. Se trata de un trabajo de abstracción de los fenómenos que se presentan siendo éstos sistemáticamente distribuídos por el pensamiento según algunas de sus propiedades o atributos, operando al mismo tiempo la existencia de esa clasificación como organizadora del pensamiento y de la visión que se tiene sobre la realidad. Es decir que la forma como se clasifican los eventos tiene una implicación fundamental sobre el modo como se representan y se interviene sobre ellos. "Las clasificaciones son producto resultante de la forma en que agrupamos los fenómenos que nos circundan y reflejan el entendimiento de sus semejanzas y de sus diferencias" (TORT, 1989).

En la medida que la epidemiología en salud mental pueda definir y precisar un nuevo objeto de estudio, tendrá que repensar y construir nuevas modalidades clasificatorias, que resulten superadoras de aquellas organizadas en torno a entidades mórbidas y que han eludido las representaciones o puntos de vista del paciente (illnes) o las significaciones socio-culturales de los grupos humanos (sicknes). La idea de que las enfermedades, incluidas las enfermedades mentales, son eventos de naturaleza individual y a-histórica y que pueden ser definidas en forma neutra y objetiva ha cristalizado, por décadas, los sistemas clasificatorios volviéndolos incuestionables.

«... através de nuevas metáforas y nuevas metonimias debe expresarse nuestro entendimiento de los procesos de salud - enfermedad, que además de los sistemas de referencia biológica desarrollen nuevos indicadores que tengan la capacidad de medir dimensiones hasta ahora no mensurables del proceso saludenfermedad» (BARRETO, 1999).

Si se ha ganado en la comprensión de la determinación social y cultural de la enfermedad y el sufrimiento psíquico, si se han acumulado importantes conocimientos sobre las heterogéneas significaciones con que los individuos y las sociedades vivencian sus problemas de salud mental, la epidemiología debe desarrollar nuevas lógicas clasificatorias que le permitan apropiarse de los avances conceptuales logrados sobre la salud mental.

\section{REFERÊNCIAS}

ANDREOLI S., ALMEIDA FILHO N.; COUTINHO E.; MARI J. Identificaçao de casos psiquiátricos em estudos epidemiológicos multifásicos : métodos, problemas e aplicabilidade. Rev Saúde Pública, 34 (5), 2000 p. 475-483.

ASOCIACIÓN AMERICANA DE PSIQUIATRÍA. Manual diagnóstico y estadístico de los trastornos mentales. Barcelona: Editorial Masson, 1995.

AUGSBURGER A. De la epidemiología psiquiátrica a la epidemiología en salud mental : el sufrimiento psíquico como categoría clave. Cuadernos Médico sociales $\mathrm{n}^{\circ}$ 81, Argentina, 2002. p. 61-75

BARRETO M. Por uma epidemiologia da saúde coletiva. Rev Brasileira de Epidemiologia. v. I $\mathrm{n}^{\mathrm{O}} 2$, 1999. p.104-122

BASAGLIA F. La institución negada. Informe de un hospital psiquiátrico. Barcelona: Barral Ediciones, 1972.

CONRAD P, SCHNEIDER J, Sobre la medicalización de la anormalidad y el control social. En: Psiquiatría Crítica. La política de la salud mental. Barcelona : Ediciones de La piqueta, 1982.

CONSORCIO INTERNACIONAL DE LA OMS EN EPIDEMIOLOGÍA PSIQUIÁTRICA. Comparación transnacional de la prevalencia de los trastornos mentales y los factores con ellos correlacionados. Boletín de la Organización Mundial de la Salud, 2000, 78(4) : 413-426.

FELDMAN N.; BAÑOS L.; ¿Porqué el diagnóstico? Revista Kaos Psicoanálisis $\mathrm{n}^{\circ}$ 3. Argentina: Homo Sapiens ediciones, 1995. p. 87-117

FREUD S. Lecciones de introducción al psicoanálisis, Conferencia 23. Obras Completas, Vol. III Barcelona : Biblioteca Nueva, 1981

FREUD S. El malestar en la cultura. Obras Completas, Vol. III Barcelona: Biblioteca Nueva, 1981

GALENDE E. De un horizonte incierto. Psicoanálisis y saud mental en la sociedad actual. Buenos Aires: Editorial Paidos, 1997.

GRIMBERG M. Sexualidad y relaciones de género: una aproximación a la problemática de la 
Augsburger; A.C. "La inclusión del sufrimiento psíquico: un desafio para la epidemiologia"

prevención al vih-sida en sectores populares de la ciudad de Buenos Aires. Cuadernos Médico sociales $\mathrm{n}^{\circ} 75$, Argentina, 1999. p. 65-76

KESSLER R. Epidemiología psiquiátrica : algunos avances recientes y futuras orientaciones. Boletín de la Organización Mundial de la Salud. 78 (4), 2000 p. 464-474.

ORGANIZACIÓN PANAMERICANA DE LA SALUD. Clasificación estadística internacional de enfermedades y problemas relacionados con la salud. V.2, Washington, 1995.

RAIMBAULT G. El psicoanálisis en las fronteras de la medicina. Barcelona: Editorial Ariel, 1985.

Ana Cecilia Augsburger es Magister en Salud Pública de la Universidad Nacional de Rosario, (Argentina). Profesora e Investigadora de la Facultad de Psicología de la Universidad Nacional de Rosario. O Endereço eletrônico da autora é: augsburgerc@yahoo.com

Ana Cecília Augsburger

La inclusión del sufrimiento psíquico: um

desafio para la epidemiologia

Recebido: 12/12/2003

$1^{\text {a }}$ revisão: $29 / 3 / 2004$

Aceite final: 18/4/2004 